\title{
On the design of an energy-harvesting protocol stack for Body Area Nano-NETworks
}

\author{
Giuseppe Piro, Gennaro Boggia, and Luigi Alfredo Grieco ${ }^{1}$ \\ DEI, Politecnico di Bari \\ Via Orabona 4, 70125, Bari, Italy
}

\begin{abstract}
Body Area Nano-NETworks (BANNETs) consist of integrated nano-machines, diffused in the human body for collecting diagnostic information and tuning medical treatments. Endowed with communication capabilities, such nanometric devices can interact with each other and the external micro/macro world, thus enabling advanced health-care services (e.g., therapeutic, monitoring, sensing, and telemedicine tasks). Due to limited computational and communication capabilities of nano-devices, as well as their scarce energy availability, the design of powerful BANNET systems represents a very challenging research activity for upcoming years. Starting from the most significant and recent findings of the research community, this work provides a further step ahead by proposing a hierarchical network architecture, which integrates a BANNET and a macroscale health-care monitoring system and two different energy-harvesting protocol stacks that regulate the communication among nano-devices during the execution of advanced nano-medical applications. The effectiveness of devised solutions and the comparison with the common flooding-based communication technique have been evaluated through computer simulations. Results highlight pros and cons of considered approaches and pave the way for future activities in the Internet of Nano-Things and nano-medical research fields.
\end{abstract}

Keywords: Body Area Nano-NETworks, energy-harvesting aware protocols,

\footnotetext{
${ }^{1}$ Corresponding author: Giuseppe Piro Authors' email: \{name.surname\}@poliba.it.
}

Preprint submitted to Nano Communication Networks Journal (Elsevier)November 10, 2014 
performance evaluation

\section{Introduction}

In upcoming years, the innovation process triggered by nanotechnologies is expected to foster the development of integrated devices with size ranging from one to few hundred of nanometers, very well suited for ICT, biomedical, 5 industrial, and military applications [1. This is sustaining the revolutionary transition from the Internet of Things (IoT) 2 to the Internet of Nano-Things (IoNT) 3 .

Some recent studies on graphene-based nanoantennas demonstrated how nano-machines can communicate each other by using electromagnetic (EM) waves in the Terahertz band, with extremely higher bit rates at the nanoscale (i.e., around some terabit/s), but with limited transmission ranges that cannot exceed few tens of millimeters [4] [5]. Accordingly, Wireless NanoSensor Networks (WNSNs), which are networks composed by a (potentially high) number of nano-machines able to communicate each other through the wireless channel, became the first concrete actualization of the IoNT concept [1]-[10].

In particular, the use of IoNT systems in the health-care domain discloses new horizons and never seen applications [8, [11]-[15. In this context, Body Area Nano-NETworks (BANNETs) represent a specific WNSN system, operating in the human body [8]: biomedical nano-devices equipped with communication capabilities, can be implanted, ingested, or worn by humans for collecting diagnostic information (e.g., the presence of sodium, glucose, and/or other ions in blood, cholesterol, as well as cancer biomarkers and other infectious agents) and tuning medical treatments (e.g., administration of insulin and other drugs through under-skin actuators) [16].

While few scientific works already started the study of some aspects related to BANNETs (see for example [11-[15]), three important issues have not been addressed with the required depth, that are:

1. How to design a lightweight protocol suite able to fit the singular require- 
ments of nano-machines?

According to [3], nano-machines cannot execute complex tasks and, as a consequence, all the solutions already conceived for the IoT domain, cannot be directly applied to BANNETs. In line with this assumption, some contributions have already proposed valuable solutions for Media Access Control (MAC) [17] 18] and routing [19] layers. However, they mainly focus on WNSNs and do not take care of requirements and constraints that typically characterize BANNETs. Simple MAC and routing protocols enabling health-care services are presented in [12] and [13], but their formulation needs to be improved for also covering additional issues described in the sequel.

2. How to deal with limited energy resources available at the nano-scale?

Without loss of generality, it should be assumed that nano machines should count for minimal power capabilities and the adoption of suitable energy harvesting mechanisms is required for ensuring a continuous availability of nano-devices in a BANNET. With the aim of increasing, as more as possible, the lifetime of nano-networks, the entire protocol stack should be designed by jointly considering energy harvesting and energy consumption processes 20]. In this regard, some significant contributions have been presented in 21]-23]. Also in this case, such works focus on WNSN and their adaptability to BANNET environments still remains an open issue.

3. How to enable the interaction between nano-environments and the rest of the world?

A BANNET could be integrated within a complex health monitoring system [8, where different monitoring devices, that communicate among them and with a remote health-care server through Low-power and Lossy Network (LLN) technologies [24]2], may also coexist. In this scenario, it is important to evaluate the interaction between devices belonging to both nano and macro domains. Even if a hierarchical architecture has been already defined in [3, it is still not clear how to properly regulate the interaction between macro and nano devices and to define the rate of 
requests coming from the macro world that ensures the right reactivity of nano-devices (subjected to computational, technological, and energy constraints) that satisfies health-care application requirements.

To provide initial answers to the aforementioned issues, this work proposes a twofold contribution. First of all, a lightweight network architecture that integrates a BANNET within a more complete health-care monitoring system is proposed. In line with the scheme presented in [3], it is able to deliver requests coming from monitoring devices to nano-machines and forward corresponding answers in the opposite direction. Then, two different energy-harvesting aware protocol stacks (composed by both MAC and routing algorithms) have been conceived. The former scheme implements an optimal routing protocol, that selects the most suitable nano-machine which the request coming from the external world are forwarded to. Such a decision is done in order maximize the overall amount of energy that will be available into the network when a new request arrives. To this end, starting from the energy model developed in [20, the routing protocol has been defined by means of an optimization problem. The latter, instead, just delivers the request to the node with the higher energy level (greedy approach). In both cases, a handshake mechanism has been implemented at the MAC layer for identifying devices available in the neighborhood and being aware about their energy level.

The effectiveness of conceived proposals have been evaluated through computer simulations by using the emerging NANO-SIM tool [25], which models electromagnetic based nano-communications within the NS-3 simulation framework. In particular, it has been evaluated the impact of the density of nanomachines forming the BANNET and of the average rate of requests coming from the macro world have on packet loss ratio, energy and device availability, and physical transmission throughput. To provide a further insight, a comparison with respect to the simple flooding approach (according to which any request is broadcasted into the network without executing any kind of initial handshake and the answer is generated by all the available devices) is reported too. 
Results show that better system performances can be achieved when energyharvesting aware techniques are used. When compared with the flooding approach, such strategies guarantee an increase in the average amount of energy available in each nano-machine (about more 60\%), a decrease of the percentage of packet losses (up to 10\%), a gain on the percentage of active nodes (ranging from $6 \%$ to $50 \%$ ), and the reduction of the physical transmission rate (up to $20 \%$ ). Moreover, they demonstrate that the proposed greedy strategy, despite its lowest computational complexity, guarantees results very close to those reached with the optimal strategy, thus becoming the best candidate for BANNETs. In authors' humble opinion this study (with particular reference to the analysis of the packet loss ratio, measured under different network conditions) could be useful to find the most suitable combination of both network size and request rate that better satisfies requirements of real nano-medical applications.

The rest of this paper is organized as follows. Sec. 2 presents a background on both IoT and IoNT paradigms, by focusing the attention on both biomedical applications and energy-harvesting techniques available at the nano-scale. Sec. 3 discusses both the conceived health-care monitoring system and the designed energy-harvesting aware protocol suites. The performance evaluation of proposed solutions is investigated in Sec. 4 . Finally, Sec. 5 draws the conclusions and discusses future activities.

\section{Background on IoT, IoNT, nano-medical applications, and energy-} related issues

In this section, a quick presentation of IoT and IoNT paradigms is proposed. Moreover, the description of main features and target applications of BANNETs, as well as energy issues affecting nano-machines diffused in the human body, is discussed too.

\subsection{Internet of Things in a nutshell}

The notion of Internet of Things (IoT) was early conceived in 1999 by Kevin Ashton [26] to mean the binding of Radio Frequency Identifiers information 
to the Internet. Soon, it became a technological paradigm enabling advanced and desired services, including monitoring and control in smart-cities, industrial plants and military environments. In a more recent form, the IoT is supposed to be capable of managing a potentially very large number of smart wireless devices forming a capillary networking infrastructure that can be connected to the Internet [27] 2] 28].

At this moment, it is widely recognized that IoT can be adopted in the health-care domain for handling a number of tasks [29], including the remote monitoring of patients [30]-33, the control of drugs [34, and the tracking of medical staff and equipments in their environment [35-37. However, all of these solutions do not go beyond the macro scale, thus leaving the nano-medicine applications completely unexplored.

\subsection{Towards the Internet of Nano-Things paradigm}

Thanks to the progress of the nanotechnology, it is now possible to design and manufacture nanoscale components able to perform simple tasks, including computing, storing, sensing, actuation, and communication [1] 38.

In this context, nano-antennas may support EM communications in two possible range of frequencies: the terahertz band and the upper part of the megahertz one. Despite the lowest bandwidth ensures the highest transmission ranges, it provides a very limited energy efficiency, which is unacceptable for nano-devices. For this reason, it is preferred to design nano-tranceivers working in the terahethz band (i.e., $0.1 \div 10.0 \mathrm{THz}$ ). The entire spectrum can range from a few hundred of gigahertz to almost $10 \mathrm{THz}$, enabling a channel capacity in the order of few terabit/s and a transmission range that cannot exceed few tens of millimeters 38. In addition, due to size and energy constraints of nano-machines, techniques based on the transmission of signals with long duration, which are typically adopted in Wireless Sensor Network (WSN) 2], cannot be used at the nano-scale. Considering the huge available bandwidth, a promising solution could consist in exchanging very short pulses spread over the entire spectrum. With Time Spread On-Off Keying (TS-OOK), a logical 
1 is transmitted by using a short pulse and a logical 0 is encoded as a silence. The only limitation to its straight usage is related to the time between two consecutive pulses, which should be kept longer than the pulse duration because the communication unit can work only with a very low duty-cycle, due to technological limitations [39]. The adoption of TS-OOK has two important advantages. Firstly, it does not require that nano-devices should be synchronized before starting the transmission of the packet. Moreover, it allows multiple users to safely share the same wireless medium; in fact, since the time between the transmission of two consecutive pulses has to be much longer than the pulse duration, several nano-devices can concurrently send sequence of pulses which are slightly time-shifted, without incurring in collisions.

A WNSN is a network architecture composed by a number of nano-devices able to communicate among them through EM waves in the THz channel. According to the [3], it can be composed by three different kind of nodes: nanonodes, nanorouters, and nanointerface. Nanonodes are tiny devices with very scarce energy, computational, and storage capabilities. They are diffused into a target area for sensing the environment. Nanorouters are, instead, nanodevices having sizes and resources larger than previous ones. They divide the whole network architecture in independent clusters and aggregate and process the information coming from nanonodes controlling their behavior by using short control messages. Finally, the nanointerface, which is the most complex node, inter-networks (acting as a gateway) the WNSN with the rest of the world.

\subsection{BANNET: when IoNT meets the nano-medicine field}

Nowadays, the monitoring of human activities through external sensor devices, which are strategically placed around the body, is highly used to provide ambient assisted living, sport training, streaming, emergency, computer vision, wearable health monitoring, sleep staging, and telemedicine services. All the network architectures designed to offer one of the aforementioned applications fall within the general term of Wireless Body Area Network (WBAN) 40].

However, recent developments in both nano and biotechnological fields made 
possible the realization of therapeutic nano-machines, operating either in inter and intra-cellular areas of the human body [41]16]. A system composed by these kind of devices is just called Body Area Nano-NETwork (BANNET). Besides the conventional molecular communication, also electromagnetic-based communications are considered as a viable technique for handling cooperative and coordinated tasks of nano-devices, efficient gathering of biosensor data, the correlation of biosensor inputs for making decisions, and the transmission of information to external entities 41. Thanks to their capability to interact with organs and tissues, BANNETs can be exploited in a very large number of pioneering nano-medical applications, which include [42] 43]:

- immune system support: a group of specialized nano-machines is diffused in the human body for protecting the organism against diseases, identifying pathogen elements, and localizing malicious agents and cells (like cancer cells);

- bio-hybrid implant: nano-devices can be used for supporting or replacing components of the human body (i.e., organs, nervous tracks, and lost tissues);

- drug delivering systems: in that case, a BANNET should be able to compensate metabolism diseases by strategically releasing chemicals (like glucose for diabetic patients) and mitigate the effect of neurodegenerative diseases by means of the timely administration and distribution of drugs in the organism;

- heath monitoring: nano-machines in the human body may collects information about the presence of specific molecules, such as oxygen and cholesterol level, measure biological functionalities of the patient, and deliver them to external monitoring devices; and genes is another domain where BANNETs can take place. 
It is very interesting to note that all the aforementioned applications rely on an heterogeneous architecture that integrates the nano-network within a more complex and distributed health-care system, also composed by external monitoring devices and remote servers. Despite some works have already proposed initial analysis on features and capabilities of such a network architecture (see for example contributions presented in [11] 12] 13, 15] 44]), more accurate studies need to be done before considering BANNETs a mature technology. In this context, network architecture, communication paradigm, energy harvesting schemes, and protocol stacks are just the first very challenging aspects that the research community is called to investigate.

\subsection{Energy consumptions and harvesting schemes for nano-networks}

In most of the cases, nanonodes should count for minimal power, data storage, processing, and communication capabilities. Hence, the jointly knowledge of energy requirements and energy harvesting mechanisms available at the nanoscale is highly important for the design of optimized BANNET architectures and for ensuring that the lifetime of nano-networks may (potentially) tend to infinity [20].

Let $E_{p}^{t x}, E_{p}^{r x}, E^{t x}(x)$, and $E^{r x}(x)$ be the energy required to transmit a 225 pulse, receive a pulse, transmit a packet of $x$ bits, and receive a packet of $x$ bits, respectively.

The study presented in [39], which considers a TS-OOK modulation scheme with pulse duration, pulse time interarrival, and transmission range equal to $100 \mathrm{fs}, 100 \mathrm{ps}$, and $10 \mathrm{~mm}$, respectively, reported that the energy required to transmit a pulse, $E_{p}^{t x}$, is equal to $1 \mathrm{pJ}$ and that the amount of energy required to receive a pulse, $E_{p}^{r x}$, is equal to $0.1 \mathrm{pJ}$ (i.e., $E_{p}^{r x}=E_{p}^{t x} / 10$ ). Moreover, by considering a packet of $x$ bits, the energy required to handle its transmission and reception are given by:

$$
E^{t x}(x)=x \cdot w \cdot E_{p}^{t x},
$$




$$
E^{r x}(x)=x E_{p}^{r x}=\frac{x}{10} E_{p}^{r x} .
$$

where the parameter $w$ in Eq. (1) describes the probability to have a symbol 1 within the stream of $x$ bits (generally, $w$ is set to 0.5 because symbols are equiprobable). In the reception process, instead, all bits brings to an energy 230 consumption.

Conventional energy harvesting mechanisms, e.g., solar energy, wind power, or underwater turbulences, cannot be applied in this context because technological limitations make theme not feasible at the nano-scale, but novel schemes should be adopted for providing energy to nano-machines [20]. At this moment, a piezoelectric nanogenerator, composed by an array of $Z n O$ nanowires, a reflecting circuit, and a ultra-nanocapacitor, represents the most promising and pioneering system to power such kind of devices. In particular, the electric current is generated between the ends of nano-wires when they are bent or compressed. In details, as soon nano-wires are released, the current is used to recharge the capacitor. The compressed-release cycle is provided by means of mechanical vibration, such as air conditioning, heartbeat, and so on [20].

An accurate model describing the energy harvesting rate of piezoelectric nanogenerators has been already developed in [20] and it will be considered for the specific purposes of the present work. It says that after $n_{c}$ compress-release cycles, the voltage of the charging capacitor, $V_{c a p}\left(n_{c}\right)$, can be computed as:

$$
V_{\text {cap }}\left(n_{c}\right)=V_{g}\left(1-e^{-\frac{n_{c} \Delta Q}{V_{g} C_{c a p}}}\right)
$$

where $C_{c a p}, \Delta Q$, and $V_{g}$ are the total capacitance of the ultra-nanocapacitor, the generator voltage, and the harvested charge per cycle, respectively. Considering technological constraints of nano-devices, typical values of such quantities are: $C_{\text {cap }}=9 \mathrm{nF}, \Delta Q=6 \mathrm{pC}$, and $V_{g}=0.42 \mathrm{~V}[20$.

The amount of accumulated energy, $E_{c a p}$, is instead expressed as:

$$
E_{c a p}\left(n_{c}\right)=\frac{1}{2} C_{c a p} V_{c a p}^{2}\left(n_{c}\right) .
$$




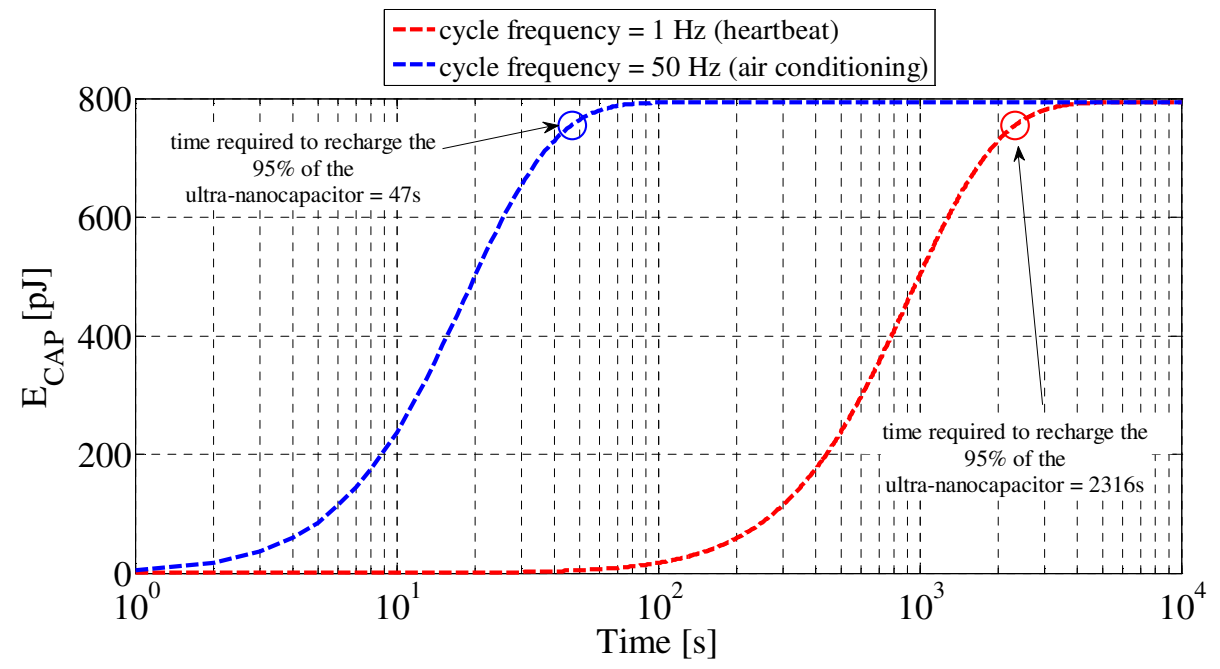

Figure 1: Energy stored into the ultra-nanocapacitor as a function of the recharging time.

The time required to recharge the ultra-nanocapacitor depends on the frequency of compressed-release cycles offered by the external vibration source, $f_{c}$. For example, for devices placed on the skin surface, vibration stimuli can be provided by the air conditioning (for which $f_{c}=50 \mathrm{~Hz}$ ). For nano-machines integrated within the human body, instead, the heartbeat represents the only energy source with $f_{c}=1 \mathrm{~Hz}[20$. Fig. 1 gives an immediate picture of this aspect by reporting the energy stored in the ultra-nanocapacitor during the time when vibration stimuli are provided by both air conditioning and heartbeat (note that curves are obtained considering the energy model presented in Sec.

255 2.4. From results it is evident that: (i) the amount of energy available for a nano-machine is always, as expected, equal to $800 \mathrm{pJ}$ and (ii) the time required to reach the $95 \%$ of the maximum energy capacity is equal to $47 \mathrm{~s}$ and $2361 \mathrm{~s}$ when the piezoelectric generators are excited by the air conditioning and the heartbeat, respectively.

This simple numerical example shows how a single nano-device diffused in the human body has very scarce energy availability: in line with Eq. (1), it can just send up to 8 packets of 200 bits each with the maximum amount of energy it 
may harvest. Moreover, the time required to recharge the nano-battery is very high, especially for those nodes diffused in the human body. It emerges that in this context it is impossible to reach the high transmission rates allowable at the terahertz channel.

In conclusion, the design of effective energy-harvesting aware protocols is extremely important for really enabling advanced health-care service, especially in scenarios with evident energy issues. Interesting contributions in this direction have already presented in literature: they consider advanced scheme for adjusting transmission settings to minimize energy consumptions [21, energy-efficient physical layer for WNSN [23, energy-harvesting MAC protocol for WNSN [18] energy and spectrum-aware MAC protocol for WNSN [22, and routing framework for energy harvesting WNSN [19]. However, none of these solutions take care of requirements and constraints of BANNETs and they do not offer complete answers to issues detailed in the introductory section. For this reason, the proposal described in the following section can be considered completely complementary to all approaches and methodologies presented so far.

\section{System architecture and energy-harvesting protocols for BANNETs}

In line with 3 11, 12,13, 15, the health-care monitoring system considered in this work is composed by a BANNET, a set of external monitoring devices, and a remote health-care server (see Fig. 2). According to a typical IoT architecture, monitoring devices communicate with the nanointerface and a network coordinator (which provides the connectivity with a remote health-care server through a wireless/wired broadband technology) by using IEEE 802.15.4 radio $2^{2}$ The interaction between macro and nano domains can be handled by means of a request/response process. In fact, it is assumed that each monitoring device is configured for tracking some biological functionalities of a given

${ }^{2}$ IEEE 802.15.4 standard is considered the most successful enabling technologies for short range low rate wireless communications and provides all the details for both MAC and PHY layers 45,26 


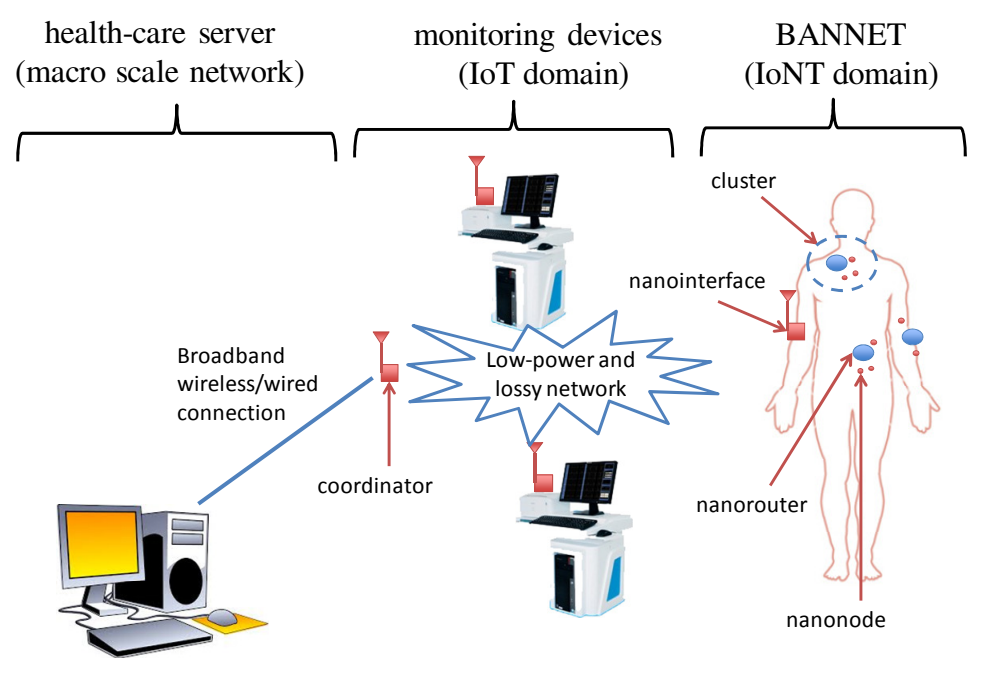

Figure 2: Complete health-care system architecture envisaged in the present work.

patient during the time. To this end, it sends specific request messages to the nanointerface of the BANNET through the IoT network infrastructure. The nanointerface will deliver the received request to all nanorouters, thus allowing them to retrieve an answer from their corresponding clusters. Then, the requests generated by a sub-set of nanonodes are sent back to the monitoring device in the opposite direction (i.e., through the reference nanorouter and the nanointerface). Finally, the monitoring devices will deliver all the collected information to the remote health-care server.

It is important to remark that the definition of the maximum number of requests per second coming from the macro domain is not easy to evaluate. Due to huge energy constraints, in fact, nanonodes may not be always able to provide an answer to external demands, especially when requests arrive too fast with respect to the time required to harvest the energy. The analysis conducted in Sec. 4 will shed some lights on this very important aspect, thus providing significant guidelines for further studies in the future.

On the other hand, the selection of the most suitable nanonode able to provide an answer to the request coming from the macro domain represents a crucial task for each nanorouter. Of course, the routing process should be 
handled in order to reduce the overall energy consumption and maximize the lifetime of the whole system. Starting from these premises, two different routing strategies, which exploit an optimal approach and a greedy scheme, respectively, have been designed. Furthermore, both of them make use of an energy-aware MAC protocol able to identify the group of nanonodes available in each cluster through a simple handshake mechanism. Note that from this moment on, the term available node is used to identify the node with energy that is able to communicate with others.

To this aim, four different messages have been defined as it follows.

- neighbor discovery message: it is broadcasted by the nanorouter for discovering, in its cluster, active nanonodes;

- energy feedback message: it is generated by the nanonode as an answer to the neighbor discovery message and contains the energy level stored within its ultra-nanocapacitor;

- request message: it carries the question generated by a monitoring device and it is forwarded by the nanointerface to all nanorouters and then sent to a given nanonode of each cluster, which has been properly selected by the routing scheme;

- answer message: it represents the answer generated by the nanonode and sent back to the nanointerface.

In what follows, $N_{d}, N_{e}, N_{r}$, and $N_{a}$ are the size, expressed in bits, of neighbor discovery, energy feedback, request, and answer messages, respectively.

\subsection{Energy-harvesting aware MAC protocol}

According to the amount of available energy, a nanonode may fall in active or idle mode: when the harvested energy is higher than a given threshold, i.e., $E^{t h}$, the node is active and can participate to the request/response mechanism; otherwise, it remains in idle mode and just continues to harvest energy. The 
energy threshold is defined as a multiple of the amount of energy required to complete the request/response mechanism, that is:

$$
E^{t h}=\alpha\left[E^{r x}\left(N_{d}\right)+E^{t x}\left(N_{e}\right)+E^{r x}\left(N_{r}\right)+E^{t x}\left(N_{a}\right)\right] .
$$

Considering node constraints discussed in [3], and in line with solutions already presented in [13] 18, it has been designed an asynchronous MAC protocol. In particular, due to the limited amount of available energy and the impossibility to guarantee time synchronization at the nano-scale, no specific frame structures in the time domain and acknowledgment strategies are implemented at the MAC layer. Every time the node receives a message from upper layers when it is in active state, it will transmit the packet through the physical interface without executing any kind of control. It is important to note that despite the absence of any channel sensing mechanism, the probability to have physical collisions is close to 0 because the time required to transmit a given packet is much smaller that propagation delay and the time interval between two consecutive transmissions.

The handshake mechanism consists of the exchange of neighbor discovery and energy feedback messages. Before sending the request message, the nanorouter broadcasts the neighbor discovery message and collects all the cor${ }_{345}$ responding answers for a time interval equal to $T=T_{N_{d}}^{t x}+T_{N_{e}}^{t x}+D^{\max }$, where $T_{N_{d}}^{t x}, T_{N_{e}}^{t x}$, and $D^{\max }$ are the transmission time of the neighbor discovery message, the transmission time of the energy feedback message, and the maximum propagation delay for a given cluster, respectively. Hence, active nanonodes will respond with an energy feedback message containing the latest estimation of the amount of energy stored within the ultra-nanocapacitor. To prevent the reception of packets generated by other nanonodes within the cluster and avoid unuseful waste of energy provided by the reception process, each nanonodes deactivates the physical reception interface for a time interval equal to $T_{N_{e}}^{t x}+D^{\max }$.

355 Once the handshake mechanism has been completed, the nanorouter runs the routing strategy for selecting the most suitable node to which forwarding 
the request coming from the external monitoring device. Hence, it generates request message containing the type of the request and the ID of the selected nanonode. Now, only the selected nanonode will generate and transmit the corresponding answer message. The others, instead, will deactivate the physical reception interface for a time interval equal to $T_{N_{a}}^{t x}+D^{\max }$, thus limiting further useless waste of energy.

\subsection{Optimal energy-harvesting aware routing protocol}

The optimal energy-harvesting aware routing protocol aims at maximizing the overall amount of energy available within each cluster of the BANNET.

Let $\lambda, t_{k}$, and $E_{i}\left(t_{k}\right)$ be the aggregate rate of requests coming from external monitoring devices, the time instant at which the nanorouter receives a request message from the nanointerface, and the latest energy level sent by the $i$-th nanonode, respectively. Moreover, let $\Delta t=1 / \lambda$ be the average time interval between the reception of two consecutive requests.

At the end of the handshake process, the nanorouter estimates the amount of energy available in its cluster, i.e., $E^{\text {tot }}\left(t_{k}\right)$, as in the following:

$$
E^{t o t}\left(t_{k}\right)=\sum_{i} E_{i}\left(t_{k}\right)
$$

Then, for each available nanonode, it evaluates the amount of energy that could be available when a new request will be received in the future, i.e., $E_{i}\left(t_{k}+\right.$ $1 / \lambda)$. This quantity is obtained by considering the latest energy level provided by the node in the past, i.e., $E_{i}\left(t_{k}\right)$, the energy consumed for receiving the request of $N_{r}$ bits from the nanorouter, i.e., $E^{r x}\left(N_{r}\right)$, the energy consumed for sending the answer of $N_{a}$ bits in the case it will be triggered for generating the data message, i.e., $E^{t x}\left(N_{a}\right)$, and the amount of energy that will be harvested during the consecutive $\Delta t$, i.e., $H_{i}\left(t_{k}\right)$, that is:

$$
\begin{aligned}
E_{i}\left(t_{k}+1 / \lambda\right) & =E_{i}\left(t_{k}\right)-E^{r x}\left(N_{r}\right)-\beta_{i} E^{t x}\left(N_{a}\right)+H_{i}\left(t_{k}\right) \\
& =E_{i}\left(t_{k}\right)-\frac{N_{r}}{10} E_{p}^{t x}-\beta_{i} N_{a} w E_{p}^{t x}+H_{i}\left(t_{k}\right) .
\end{aligned}
$$

where the binary parameter $\beta_{i}$ in the previous equation is set to 1 in the case the $i$-th node is selected as the destination of the request, 0 otherwise. 
The computation of $H_{i}\left(t_{k}\right)$ is complex due to the nonlinearity characterizing the energy harvested model presented in [20]. Let $\bar{n}_{c, i}$ be the number of charging cycles required to reach, under ideal conditions (i.e., by assuming an ultrananocapacitor initially empty and the absence of any kind of interruption during the charging process), the energy level stored by the $i$-th node at the end of the request/response mechanism. It can be obtained by solving Eq. (4):

$$
\bar{n}_{c, i}=-\frac{V_{g} C_{c a p}}{\Delta Q} \ln \left(1-\sqrt{\frac{2\left[E_{i}\left(t_{k}\right)-\left(N_{r} / 10+\beta_{i} N_{a} w\right) E_{p}^{t x}\right]}{C_{c a p} V_{g}^{2}}}\right) .
$$

Hence, since $f_{c} / \lambda$ represents the number of charging cycles between two consecutive requests, $H_{i}\left(t_{k}\right)$ can be evaluated as:

$$
\begin{gathered}
H_{i}\left(t_{k}\right)=E_{c a p}\left(\bar{n}_{c, i}+f_{c} / \lambda\right)-E_{i}\left(t_{k}\right)-\left(N_{r} / 10+\beta_{i} N_{a} w\right) E_{p}^{t x} \\
=\frac{1}{2} C_{c a p} V_{c a p}^{2}\left(\bar{n}_{c, i}+\frac{f_{c}}{\lambda}\right)-E_{i}\left(t_{k}\right)-\left(N_{r} / 10+\beta_{i} N_{a} w\right) E_{p}^{t x} .
\end{gathered}
$$

With the aim of making as longer as possible the lifetime of the BANNET, the routing protocol should select the $i$-th nano-device in order to ensure that the total energy level available after a time interval equal to $f_{c} / \lambda$ will be maximized. In other words, the following condition should be satisfied:

$$
\max _{i}\left\{E^{t o t}\left(t_{k}+1 / \lambda\right)\right\}
$$

Substituting Eqs. (7) and (9) in Eq. (10), the maximization problem can be easily formulated as:

$$
\max _{i}\left\{\frac{1}{2} C_{c a p} \sum_{i} V_{c a p}^{2}\left(\bar{n}_{c, i}+f_{c} / \lambda\right)\right\}
$$

\subsection{Greedy energy-harvesting aware routing protocol}

The optimal routing strategy described in the previous sub-section cannot be easily implemented by a nanorouter because of the high computational cost it requires. For this reason, a greedy scheme, which make use of a very low complex decision scheme, has been also conceived.

Similarly to the optimal algorithm, also the greedy energy-harvesting aware routing protocol is executed at the end of the handshake mechanism for selecting 

external monitoring device. In this case, however, the algorithm just selects the node with the highest energy level as the destination of the request.

\section{Performance evaluation}

The performance of the devised BANNET architecture and the behavior of conceived protocol stacks under different network conditions have been evaluated through computer simulations, carried out by using the emerging NANOSIM simulator (i.e., an open source tool modeling WNSNs and electromagnetic based communications in the terahertz channel [25]12]13]). The comparison with respect to a simple flooding mechanism (where the nanorouter broadcasts each request into the network without executing any kind of initial handshake and the answer is generated by all the available devices), which is nowadays considered a comparison scheme in the context of nano-networks [13] 47], is evaluated too.

The considered BANNET architecture is composed by one nanointerface, 10 nanorouter positioned along the arm of the patient at a mutual distance of 30 $\mathrm{mm}$, and a variable number of nanonodes (i.e., from 50 to 150 devices in each cluster). Whereas both nanointerface and nanorouters maintain a fixed position during the time, nanonodes move along the artery following the direction of the blood at the speed of $20 \mathrm{~cm} / \mathrm{s}$ [8].

In line with [39, at the physical layer a TS-OOK configuration is used with pulse duration, pulse time interarrival, and transmission range equal to $100 \mathrm{fs}$, $100 \mathrm{ps}$, and $10 \mathrm{~mm}$, respectively. The length of messages exchanged within the BANNET are set as in the following: $N_{d}=N_{e}=48$ bits; $N_{r}=N_{a}=176$ bits. Finally, the health-care monitoring system has been configured in order to generate requests through a Poisson distribution with parameter $\lambda$ chosen in the range $[0.05-3]$ request $/ \mathrm{s}$.

All tests have been conducted in order to study the impact that both the rate of requests coming from the external monitoring devices and the density 
of nanonodes have on the system behavior, evaluated by means the average amount of energy available in each device, the percentage of active nanonode, the packet loss ration experienced at the application layer, and the aggregate physical transmission rate measured within each cluster. Reported results have been averaged over 60 runs in order to minimize the impact of statistical fluctuations. The $95 \%$ confidence interval has been also computed by using the ${ }_{415}$ Gaussian statistic. However, it has not been included in all the graphs because its value is lower than the marker size.

The first important result that is presented below is the average amount of energy stored within each nano-machine (see Fig. 3). As expected, when the request rate increases, the available energy decreases as well because nanonodes consume more energy for satisfying an higher number of requests. On the other hand, the higher is the number of nodes in each cluster, the lower is the average amount of energy stored within the ultra-nanocapacitor. If the density of nanonodes increases, in fact, the probability to have an higher number of active devices within the transmission range of the nanorouter increases as well; as soon the number of satisfied requests raises, the available energy is hence reduced by transmission and reception mechanisms. Nevertheless, the most important finding is that the flooding strategy provides the highest energy consumption because it does not control the generation of answer messages within clusters of the BANNET. On the other hand, instead, the proposed approaches are able to save more energy thanks to the proper selection of the nanonode to which forwarding the request.

Since the energy level stored within the ultra-nano capacitor defines the availability of nanonodes, the percentage of active devices in each cluster just follows the same trend of the results reported above. As depicted in Fig. 4 . the amount of active nodes decreases with both request rate and network size. Also in this case, it is easy to observe how both optimal and greedy approaches presented in this paper outperform the flooding strategy by offering, in all the considered scenarios, the highest percentage of active devices.

The packet loss ratio, reported in Fig. 5 , is strictly influenced by both the 


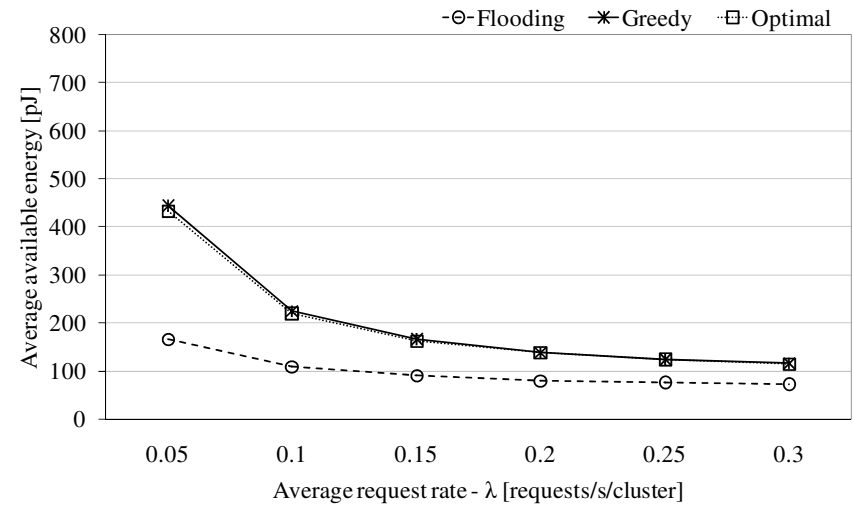

(a)

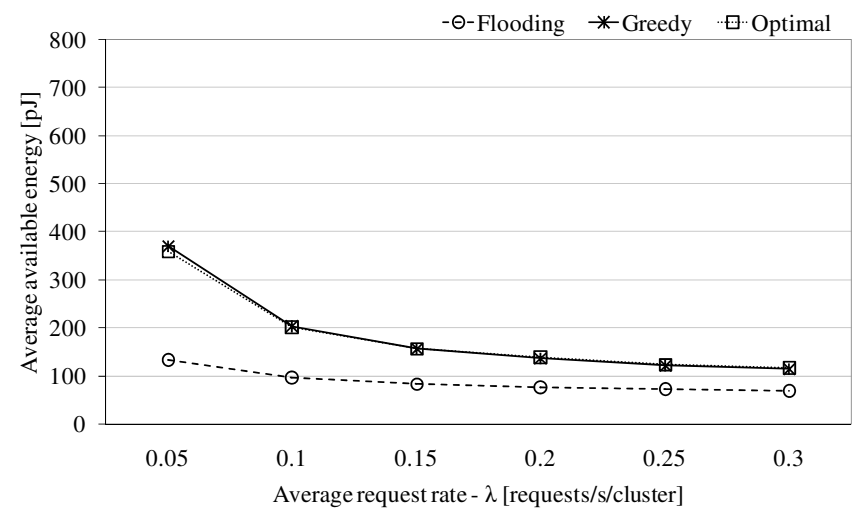

(b)

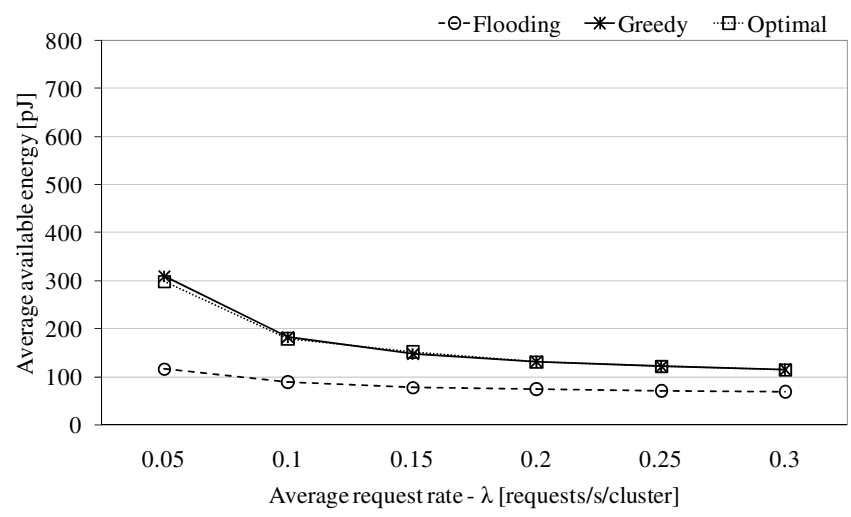

(c)

Figure 3: Average amount of energy available for each nano-device when the average number of nano-machines in each cluster is equal to: (a) 50, (b) 100, and (c) 150. 


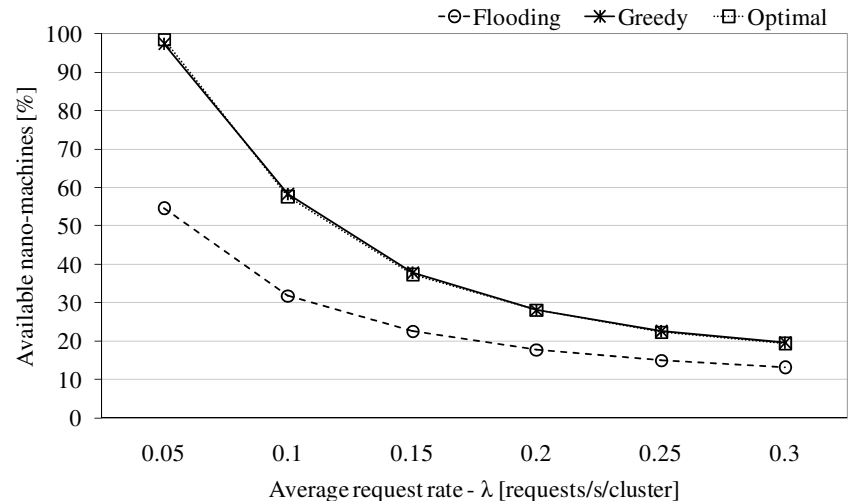

(a)

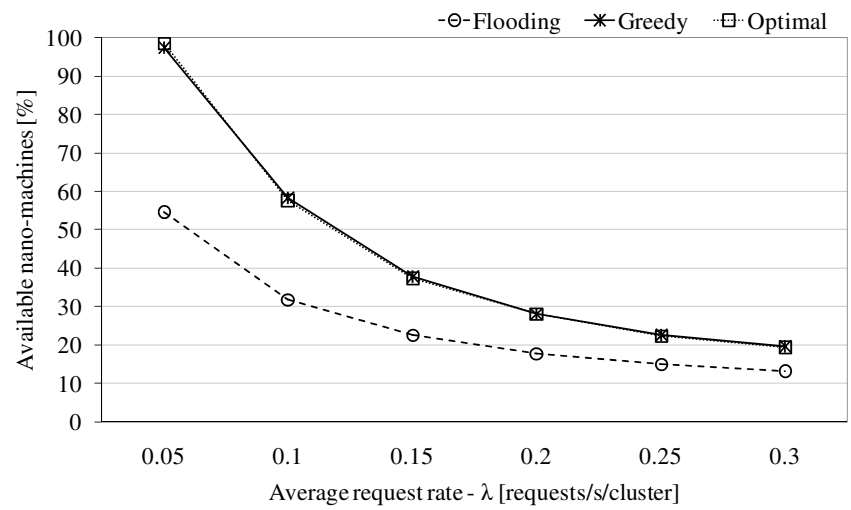

(b)

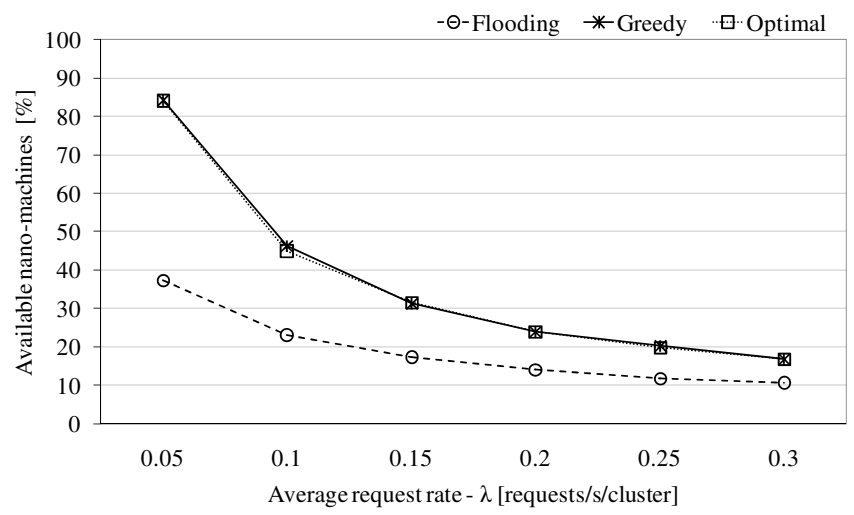

(c)

Figure 4: Percentage of available nano-nodes within a cluster of the BANNET when the average number of nano-machines in each cluster is equal to: (a) 50, (b) 100, and (c) 150. 
number of nodes into the network and the rate of requests coming from the external world. From one side, it notably grows when the rate of requests increases. This is due to the fact that nodes consume more energy for satisfying an higher number of requests and the energy harvesting rate is not enough high to recover, in time, the wasted energy. On the other hand, the number of satis-

45 fied requests increases with the number of nanonodes. As expected, in fact, the higher number of devices brings to an increment of the probability to have more active nodes within the transmission range of the nanorouter. Furthermore, the most important finding achieved in our analysis is that the conceived energyharvesting aware protocol stacks guarantee, always, the lowest percentage of packet losses at the application layer, thus ensuring the best behavior of the whole monitoring system.

The final analysis is dedicated to the transmission rate measured at the physical layer in each cluster. As reported in Fig. 6, it raises with the number of packets exchanged into the network (that increases with the request rate and 455 the number of nanonodes, as well as experiences an evident growth when the flooding approach is used) and always reaches a value in the range [40-160] bps, which is very far from the rate expected for the typical communications in the terahertz channel (i.e., tens of terabit per second).

To conclude, the conducted study clearly demonstrates the performance gain offered by energy-harvesting aware strategies. In summary, when compared with the flooding approach, such strategies guarantee an increase of the average amount of energy available in each nano-machine (about more 60\%), a decrease of the percentage of packet loss (up to 10\%), a gain on the percentage of active nodes ranging from $6 \%$ to $50 \%$, and the reduction of the physical transmission rate (up to 20\%). Moreover, the presented analysis also shows how the greedy approach, despite its very low complexity, ensures results close to those reached by the optimal strategy. Finally, reported results can be very useful for sizing the health-care monitoring system (in terms of request rate and network size) according to nano-medical application requirements (expressed, for example, in terms of percentage of satisfied requests). 


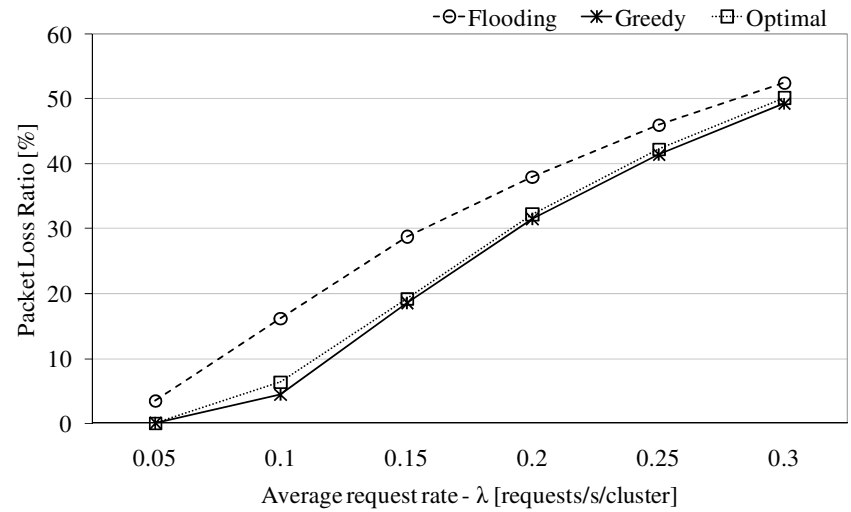

(a)

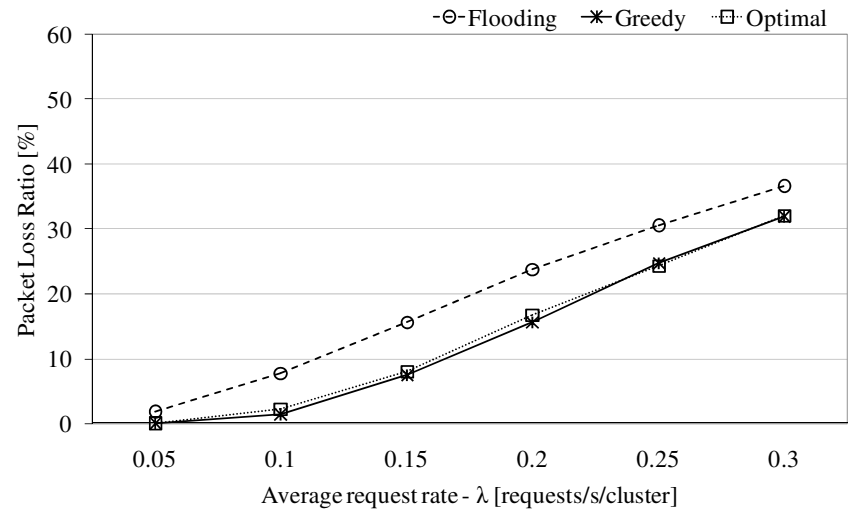

(b)

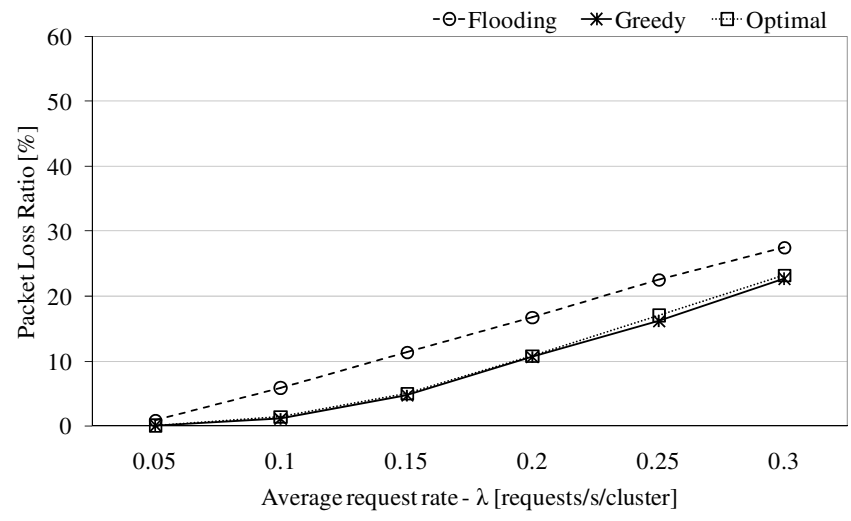

(c)

Figure 5: Packet loss ratio measured within a cluster of the BANNET when the average number of nano-machines in each cluster is equal to: (a) 50, (b) 100, and (c) 150 . 


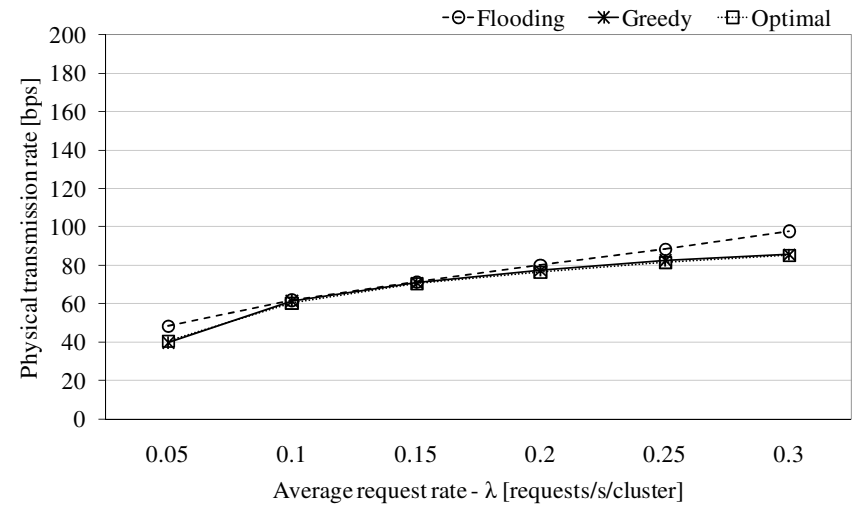

(a)

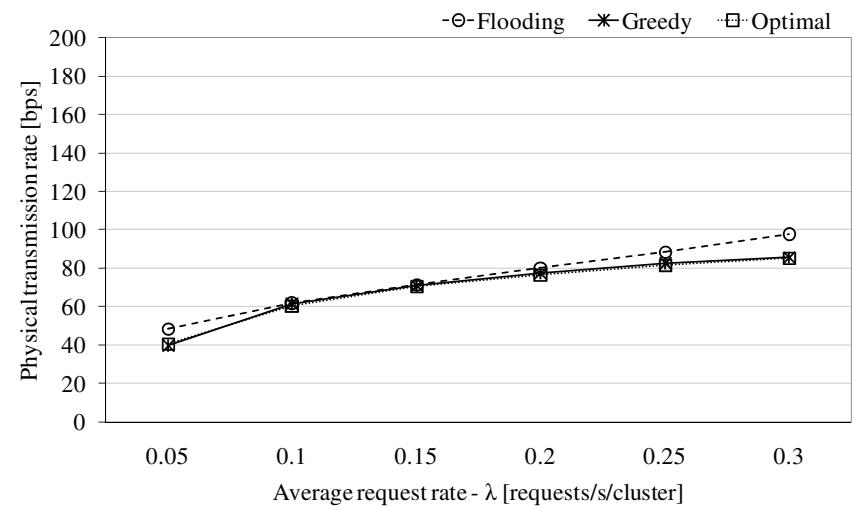

(b)

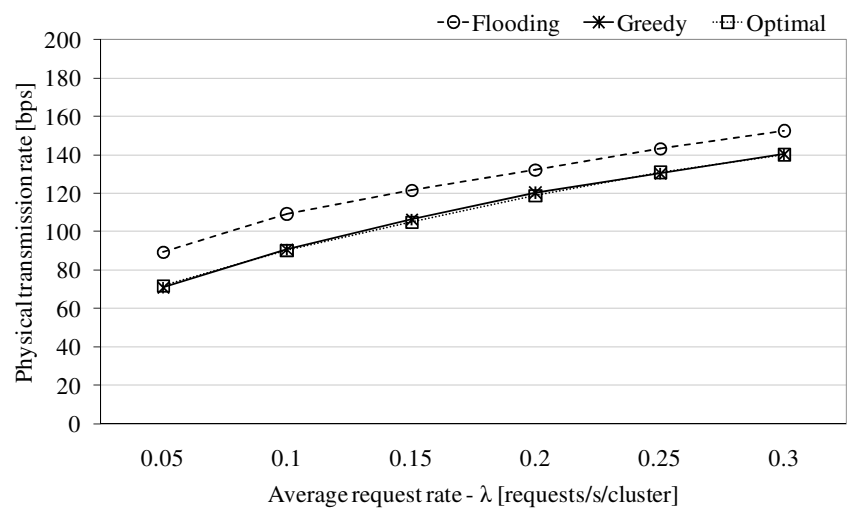

(c)

Figure 6: Physical transmission rate measured within a cluster of the BANNET when the average number of nano-machines in each cluster is equal to: (a) 50, (b) 100, and (c) 150. 


\section{Conclusions and future works}

In this paper, it has been described a hierarchical network architecture enabling a strict interaction among a Body Area Nano-NETwork (deployed into the human body) and external monitoring devices communicating among them and with the BANNET itself with classical IoT protocols. Two different energyharvesting aware protocol stacks have been deployed for properly handling the communication within the nanonetwork. Finally, performances of conceived solutions have been evaluated through computer simulations. Obtained results demonstrated that the scarce amount of energy available at the nano-scale, as well as the very low energy harvesting rate ensured within the human body, really limit the amount of data that can be exchanged within a BANNET during the execution of advanced nano-medical applications. At the same time, both the number of requests coming from the external monitoring devices and the network size significantly influence the behavior of the entire system. The presented study has shown the advantages arising from the adoption of energyharvesting aware strategies. However, the handshake mechanism involves a non negligible energy consumption, thus preventing to reach an effective and big performance gain with respect to the simple flooding mechanism. Starting from the knowledge of identified issues, future research activities will optimize the behavior of conceived solutions and investigate their performances in more complex and realistic nano-medical scenarios.

\section{Acknowledgments}

This work was supported by the PON projects (RES NOVAE, DSS-01-02499 and EURO6-01-02238) funded by the Italian MIUR and by the European Union (European Social Fund).

\section{References}

[1] I. Akyildiz, J. Jornet, Electromagnetic wireless nanosensor networks, Nano Communication Networks 1 (1) (2010) 3-19. 
[2] M. Palattella, N. Accettura, X. Vilajosana, T. Watteyne, L. Grieco, G. Boggia, M. Dohler, Standardized protocol stack for the internet of (important) things, Communications Surveys Tutorials, IEEE 15 (3) (2013) 1389-1406.

[3] I. Akyildiz, J. Jornet, The internet of nano-things, IEEE Wireless Communicationations 17 (6) (2010) $58-63$.

[4] J. Jornet, I. Akyildiz, Graphene-based nano-antennas for electromagnetic nanocommunications in the terahertz band, in: Proc of European Conf. on Antennas and Propagation, EUCAP, 2010, pp. 1 -5.

[5] I. Llatser, C. K. D.N., C. J.M., Jornet, M. Lemme, A. Cabellos-Aparicio, E. Alarcon, Characterization of graphene-based nano-antennas in the terahertz band, in: Proc of European Conf. on Antennas and Propagation, EUCAP, 2012, pp. $194-198$.

[6] S. Balasubramaniam, J. Kangasharju, Realizing the internet of nano things: Challenges, solutions, and applications, Computer 46 (2) (2013) 62-68.

[7] J. Jornet, I. Akyildiz, The internet of multimedia nano-things, Nano Communication Networks 3 (4) (2012) $242-251$.

[8] I. F. Akyildiz, J. M. Jornet, C. Han, Terahertz band: Next frontier for wireless communications, Physical Communication 12 (2014) 16-32.

[9] S. Abadal, E. Alarcn, A. Cabellos-Aparicio, M. Lemme, M. Nemirovsky, Graphene-enabled wireless communication for massive multicore architectures, IEEE Com. Mag. 51 (11) (2013) 137-143.

[10] G. Piro, S. Abadal, A. M. Sugranes, J. Sol-Pareta, E. Alarcon, L. A. Grieco, G. Boggia, Initial mac exploration for graphene-enabled wireless networkson-chip, in: Proc. of ACM International Conference on Nanoscale Computing and Communication (NANOCOM), Atlanta, Georgia, USA, 2014.

[11] N. Agoulmine, K. Kim, S. Kim, T. Rim, J.-S. Lee, M. Meyyappan, Enabling 525 communication and cooperation in bio-nanosensor networks: toward inno- 
vative healthcare solutions, IEEE Wireless Communications 19 (5) (2012) $42-51$.

[12] G. Piro, L. A. Grieco, G. Boggia, P. Camarda, Simulating Wireless Nano Sensor Networks in the NS-3 platform, in: in Proc. of Workshop on Performance Analysis and Enhancement of Wireless Networks, PAEWN, Barcelona, Spain, 2013.

[13] G. Piro, L. A. Grieco, G. Boggia, P. Camarda, Nano-sim: simulating electromagnetic-based nanonetworks in the network simulator 3, in: Proc. of Workshop on NS- 3 (held in conjunction with SIMUTools 2013), Cannes, France, 2013.

[14] S. Misra, N. Islam, J. Mahapatro, J. Rodrigues, Green wireless body area nanonetworks: Energy management and the game of survival, IEEE Journal of Biomedical and Health Informatics, 18 (2) (2014) 467-475.

[15] Y. Chen, P. Kosmas, Towards an integrated medical imaging-sensingtherapeutic platform using electromagnetically-controlled nanomachines: A preliminary conceptual design, in: Proc. of Int. Symposium on Applied Sciences in Biomedical and Communication Technologies (ISABEL), 2011, pp. 81:1-81:5.

[16] G. Santagati, T. Melodia, L. Galluccio, S. Palazzo, Medium access control and rate adaptation for ultrasonic intrabody sensor networks, IEEE/ACM Transactions on Networking PP (99) (2014) 1-1.

[17] J. M. Jornet, J. C. Pujol, J. S. Pareta, PHLAME: A Physical Layer Aware MAC protocol for Electromagnetic nanonetworks in the Terahertz Band, Nano Communication Networks 3 (1) (2012) $74-81$.

550 [18] S. Mohrehkesh, M. C. Weigle, RIH-MAC: Receiver-Initiated Harvestingaware MAC for NanoNetworks, in: Proc. of the ACM Int. Conf. on Nanoscale Computing and Communication (NANOCOM), Atlanta, GA, 2014. 
[19] M. Pierobon, J. M. Jornet, N. Akkari, S. Almasri, I. F. Akyildiz, A routing framework for energy harvesting wireless nanosensor networks in the terahertz band, Wireless Networks 20 (5) (2014) 1169-1183.

[20] J. Jornet, I. Akyildiz, Joint energy harvesting and communication analysis for perpetual wireless nanosensor networks in the terahertz band, IEEE Transactions on Nanotechnology 11 (3) (2012) 570-580.

[21] S. Mohrehkesh, M. C. Weigle, Optimizing communication energy consumption in perpetual wireless nanosensor networks, in: Proc. of IEEE Global Communications Conference (GLOBECOM), 2013, pp. 545-550. doi:10.1109/GLOCOM.2013.6831128

[22] P. Wang, J. M. Jornet, M. G. Abbas Malik, N. Akkari, I. F. Akyildiz, Energy and spectrum-aware mac protocol for perpetual wireless nanosensor networks in the terahertz band, Ad Hoc Netw. 11 (8) (2013) 2541-2555.

[23] K. Chi, Y.-h. Zhu, X. Jiang, V. C. Leung, Energy-efficient prefix-free codes for wireless nano-sensor networks using ook modulation, IEEE Transactions on Wireless Communications, 13 (5) (2014) 2670-2682.

[24] 802.15.4e-2012: IEEE Standard for Local and Metropolitan Area Networks - Part 15.4: Low-Rate Wireless Personal Area Networks (LR-WPANs) Amendment 1: MAC Sublayer (16 April 2012).

[25] G. Piro, Nano-sim - The open source framework for simulating EM-based WNSNs, [OnLine] Available: http://telematics.poliba.it/nano-sim.

[26] K. Ashton, That "Internet of Things" Thing, RFiD Journal 22 (2009) 97114.

[27] O. Hersent, D. Boswarthick, O. Elloumi, The Internet of Things: Key Applications and Protocols, 2nd Edition, Wiley, 2012.

[28] N. Accettura, G. Piro, Optimal and Secure Protocols in the IETF 6TiSCH communication stack, in: Proc. of IEEE International Symposium on Industrial Electronics (ISIE), 2014. 
[29] L. A. Grieco, A. Rizzo, S. Colucci, S. Sicari, G. Piro, D. Di Paola, G. Boggia, IoT-aided robotics applications: technological implications, target domains and open issues, Elsevier Computer Communication.

585 [30] H. Furtado, R. Trobec, Applications of wireless sensors in medicine, in: International Convention MIPRO, 2011, pp. 257-261.

[31] R. S. H. Istepanian, A. Sungoor, A. Faisal, N. Philip, Internet of m-health things "m-iot", in: IET Seminar on Assisted Living, 2011, pp. 1-3.

[32] S. Forsstrom, T. Kanter, O. Johansson, Real-Time Distributed SensorAssisted mHealth Applications on the Internet-of-Things, in: IEEE Int. Conf. on Trust, Security and Privacy in Computing and Communications, TrustCom, 2012, pp. 1844-1849.

[33] A. J. Jara, M. A. Zamora-Izquierdo, A. F. Skarmeta, Interconnection Framework for mHealth and Remote Monitoring Based on the Internet of Things, IEEE Journal on Selected Areas in Communications 31 (9) (2013) $47-65$.

[34] A. Jara, A. Alcolea, M. Zamora, A. Skarmeta, M. Alsaedy, Drugs interaction checker based on iot, in: Internet of Things (IOT), 2010, pp. 1-8.

[35] A. Dohr, R. Modre-Opsrian, M. Drobics, D. Hayn, G. Schreier, The Internet of Things for Ambient Assisted Living, in: IEEE Int. Conf. on Information Technology: New Generations, ITNG, 2010, pp. 804-809.

[36] A. Vilamovska, E. Hattziandreu, R. Schindler, C. V. Oranje, H. D. Vries, J. Krapelse, in: RAND Europe, 2009.

[37] A. Shirehjini, A. Yassine, S. Shirmohammadi, Equipment location in hospitals using rfid-based positioning system, IEEE Transactions on Information Technology in Biomedicine 16 (6) (2012) 1058-1069.

[38] J. M. Jornet, I. F. Akyildiz, Femtosecond-long pulse-based modulation for terahertz band communication in nanonetworks, IEEE Trans. on Commun. 62 (5) (2014) 1742-1754. 
[39] J. Jornet, I. Akyildiz, Information capacity of pulse-based wireless nanosensor networks, in: Proc. of IEEE Conf. on Sensor, Mesh and Ad Hoc Communications and Networks, SECON, 2011, pp. $80-88$.

[40] S. Movassaghi, M. Abolhasan, J. Lipman, D. Smith, A. Jamalipour, Wireless body area networks: A survey, IEEE Communications Surveys Tutorials 16 (3) (2014) 1658-1686.

[41] B. Atakan, O. Akan, S. Balasubramaniam, Body area nanonetworks with molecular communications in nanomedicine, IEEE Communications Magazine 50 (1) (2012) 28-34.

[42] T. Nakano, M. Moore, F. Wei, A. Vasilakos, J. Shuai, Molecular communication and networking: Opportunities and challenges, IEEE Transactions on NanoBioscience 11 (2) (2012) 135-148.

[43] I. F. Akyildiz, F. Brunetti, C. Blázquez, Nanonetworks: A new communication paradigm, Comput. Netw. 52 (12) (2008) 2260-2279.

[44] K. Kumar, Human health monitoring mobile phone application by using the wireless nanosensor based embedded system, in: International Conference on Information Communication and Embedded Systems (ICICES), 2013, pp. $889-892$.

[45] IEEE std. 802.15.4, Part 15.4: Low-Rate Wireless Personal Area Networks (LR-WPANs) (16 June 2011).

[46] S. Sciancalepore, G. Piro, E. Vogli, G. Boggia, L. A. Grieco, On securing IEEE 802.15.4 networks through a standard compliant framework, in: EuroMed Telco Conference 2014, Naples, IT, 2014.

[47] B. Atakan, O. B. Akan, Carbon nanotube-based nanoscale ad hoc networks, IEEE Communications Magazine 48 (6) (2010) $129-135$.

[48] D. G. Cramp, E. R. Carson, The Circulatory System, Taylor \& Francis, 1986. 